\title{
Electron transfer in zinc-reconstituted nitrite reductase from Pseudomonas aeruginosa
}

\author{
Andrea BELLELLI + , Peter BRZEZINSKI $\dagger$, Marzia ARESE*, Francesca CUTRUZZOLÀ ${ }^{\star}$, Maria Chiara SILVESTRINI ${ }^{\star}$ \\ and Maurizio BRUNORI \\ *Department of Biochemical Sciences and C.N.R. Center of Molecular Biology, University of Rome 'La Sapienza', P.za Aldo Moro 5, 00185 Roma, Italy and \\ $\dagger$ †epartment of Biochemistry and Biophysics, Chalmers University of Technology and University of Goteborg, Medicinaregatan 9C, S-413 90, Goteborg, Sweden
}

1. The catalytic cycle of the haem-containing nitrite reductase (NIR) from Pseudomonas aeruginosa involves electron transfer between the two prosthetic groups of the enzyme, the c-haem and the $\mathrm{d}_{1}$-haem; this reaction was shown to be slow by stopped-flow analysis. The recombinant enzyme, expressed in Pseudomonas putida, contains the c-haem but no $\mathrm{d}_{1}$-haem; we have reconstituted this protein with Zn-protoporphyrin IX in the place of the $\mathrm{d}_{1}$-haem. 2. Photoexcitation of Zn-NIR is followed by electron transfer from the triplet excited state of the $\mathrm{Zn}$-porphyrin to the oxidized c-haem, with a rate constant of $7 \times 10^{5} \mathrm{~s}^{-1}$; since the intermediate with reduced c-haem is not significantly populated, we conclude that the back reaction is probably as fast. 3 . Even taking into account that in the native NIR the driving force is close to zero, the rate constant for the $\mathrm{c} \rightarrow \mathrm{d}_{1}$ electron transfer, estimated from our experiments, is still much higher than that observed by stopped flow $\left(k=0.3 \mathrm{~s}^{-1}\right)$ using reduced azurin as the electron donor. This finding may be a direct kinetic indication that reduction of the $d_{1}$-haem is associated with a substantial reorganization of the co-ordination of the metal, as shown by spectroscopy of the oxidized and reduced NIR.

\section{INTRODUCTION}

Nitrate and nitrite, the toxic oxidized states of nitrogen, are metabolized by denitrifying bacteria which use them as electron donors to yield as waste products reduced nitrogen compounds such nitric or nitrous oxide $\left(\mathrm{NO}\right.$ or $\left.\mathrm{N}_{2} \mathrm{O}\right)$ and even $\mathrm{N}_{2}$.

The reduction of $\mathrm{NO}_{2}{ }^{-}$to $\mathrm{NO}$ is catalysed by nitrite reductases (NIRs), metalloenzymes which use either copper or haem iron; haem NIRs show a very peculiar property since, besides reducing nitrite to NO in a one-electron reaction, they also reduce oxygen to water, in a reaction involving four electrons [1]. An interesting mechanism for these two reactions has been recently proposed by Fulop et al. [2], on the basis of the high-resolution threedimensional structure of the haem-containing NIR from Thiosphaera pantotropha.

Haem-containing NIR from Pseudomonas aeruginosa is a homodimeric protein with a molecular mass of $120 \mathrm{kDa}$; each monomer contains one covalently bound c-type haem and one weakly bound $d_{1}$-type haem (for the structure of the $d_{1}$-haem see [3]; reviewed in [4]). The crystallographic structure of the oxidized form of the related haem-containing NIR from Thiosphaera pantotropha [2] shows that the haems are bound to two distinct domains in each monomer, forming an angle of $60^{\circ}$ with respect to each other; the iron-to-iron distance is $20.6 \AA$, in agreement with the electron paramagnetic coupling between the iron atoms observed in NIR from Ps. aeruginosa [5,6]. The intramolecular c $\Leftrightarrow \mathrm{d}_{1}$ electron transfer (eT) is rather slow when the electron donor/acceptor is azurin, the $\mathrm{c} \rightarrow \mathrm{d}_{1}$ eT rate constant being $0.3 \mathrm{~s}^{-1}$ [7]; however, when the reduced enzyme is mixed with $\mathrm{O}_{2}$, the rate constant for c-haem oxidation is $\geqslant 200 \mathrm{~s}^{-1}$, being synchronous with oxygen binding [8].

Zn-containing porphyrins can be photoexcited to populate a long-lived triplet state [9], which may be able to transfer an electron to other redox centres (see, for example, $[10,11]$ ). This technique has been recently exploited to measure the eT rate in a complex of Zn-cytochrome $c$ with (beef heart) cytochrome $c$ oxidase [12]. In this study we show that recombinant apo-NIR from Ps. aeruginosa (which contains only the c-haem [13]) can be reconstituted with $\mathrm{Zn}$-protoporphyrin IX, which is bound to the $\mathrm{d}_{1}$-haem binding site. This hybrid Fe-Zn-NIR (herein called ZnNIR) has been purified and characterized. Following rapid laser photoexcitation, the triplet excited state of the Zn-porphyrin in the pocket of the $d_{1}$-haem decays rapidly by eT to the oxidized c-haem; the electron is then back transferred to the Zn-porphyrin ${ }^{+}$. The data are discussed with reference to the threedimensional structure of the enzyme and to the eT rates obtained by conventional methods.

\section{MATERIALS AND METHODS}

Recombinant NIR was obtained from cultures of Ps. putida as described previously [13]; the enzyme synthesized by this microorganism lacks the $\mathrm{d}_{1}$-haem, and is herein called apo-NIR.

Apo-NIR was incubated with two stoichiometric excesses of Zn-porphyrin (Sigma Chemical Co., St. Louis, MO, U.S.A.) in $0.1 \mathrm{M}$ Tris $/ \mathrm{HCl}$ buffer, $\mathrm{pH} 8.0$, and the extent of reconstitution was checked spectrophotometrically using a Varian Cary-219 spectrophotometer; after $2 \mathrm{~h}$ incubation at $4{ }^{\circ} \mathrm{C}$, the solution of Zn-NIR was eluted through a Sephadex G25 column (Pharmacia, Uppsala, Sweden) to remove the excess Zn-porphyrin. After extensive dialysis against $10 \mathrm{mM}$ Tris $/ \mathrm{HCl}$ buffer, $\mathrm{pH} 8.0$, containing $2 \mathrm{mM}$ sodium citrate, the amount of zinc and iron was determined by atomic absorption spectroscopy, which showed that the $\mathrm{Zn}$-porphyrin was bound in excess (in one case up to $1: 1.8)$ with respect to the $\mathrm{d}_{1}$-haem sites.

Abbreviations used: NIR, nitrite reductase; apo-NIR, recombinant NIR lacking the $d_{1}$-haem; Zn-NIR, recombinant NIR in which the $d_{1}$-haem has been replaced with Zn-protoporphyrin IX (each monomer contains one Fe c-haem and one Zn-porphyrin); ANS, 1-anilino-8-naphthalenesulphonate; eT, electron transfer. Time constants are defined as the reciprocal of the rate constants; notice that all processes observed in this study are first order.

* To whom correspondence should be addressed. 
The apo-NIR was titrated with 1-anilino-8-naphthalenesulphonate (ANS) (Sigma Chemical Co., St. Louis, MO, U.S.A.) using a Spex (Edison, NJ, U.S.A.) Fluoromax spectrofluorimeter; other fluorescence measurements, as well as the lifetime of the $\mathrm{Zn}$-NIR, were carried out using a phase-modulated Spex spectrofluorimeter model 1680.

Measurements of photoinduced eT were carried out using an apparatus of local design [14]; briefly the $8 \mathrm{~ns}$ pulse at $532 \mathrm{~nm}$ obtained from a NdYAG laser (Spectra Physics model GCR190) was focused on to a four-window quartz cuvette sealed to a modified Thunberg tube containing $2 \mathrm{ml}$ of a $12 \mu \mathrm{M}$ (monomer) solution of Zn-NIR in the desired buffer. The transmittance of the sample was monitored using a $250 \mathrm{~W}$ halogen lamp (oriented at $90^{\circ}$ with respect to the laser) and a photomultiplier tube (Hamamatsu R269 or R712) connected to a current-to-voltage converter (Hamamatsu $\mathrm{C} 1053$, bandwith $3 \mathrm{MHz}$ ) and finally to a digital oscilloscope (Nicolet, model 490). The power of the laser was tuned in order to minimize the photochemical damage of the sample (usually to one-tenth of its maximal power of $400 \mathrm{~mJ}$ per pulse); since a satisfactory signal-to-noise ratio requires averaging of up to 500 traces, in a typical experiment carried out at several wavelengths the same sample stands over 10000 flashes; nonetheless, the properties of the sample were unchanged at the end of the experiment.

The data were analysed using either commercial software (mostly MATLAB, by The Math Works Inc., South Natick, MA, U.S.A.) or programs developed for the specific purpose using the Borland Pascal Compiler (Borland, Scotts Valley, CA, U.S.A.).

\section{RESULTS}

\section{Preparation and reconstitution of apo-NIR with Zn-protoporphyrin IX}

The expression of NIR from Ps. aeruginosa in the heterologous system of Ps. putida has already been described [13]; in this micro-organism the recombinant NIR is expressed as a soluble dimeric semi-apoprotein, containing one c-haem per monomer, but lacking the $\mathrm{d}_{1}$-haems; addition of $\mathrm{d}_{1}$-haem yields an enzyme indistinguishable from the native Ps. aeruginosa NIR. This is therefore an ideal starting material for reconstitution with different haems.

Reconstitution of Zn-NIR was carried out on a larger scale by incubation of the apo-NIR with a 2-fold excess of the $\mathrm{Zn}$ porphyrin, as described in the Materials and methods section. Binding of the Zn-porphyrin at the $\mathrm{d}_{1}$-haem empty pocket is strongly suggested by (i) the competition with ANS (see below), and (ii) the observation that upon reconstitution with $\mathrm{Fe}$ protoporphyrin IX, eT to the c-haem is observed (results not shown, see also [15]).

The c-haem of $\mathrm{Zn}-\mathrm{NIR}$, which is in the reduced state after reconstitution, is fully oxidized by addition of a slight stoichiometric excess of sodium ferricyanide; the reaction is reversible and the ferrous c-haem is easily obtained by addition of dithionite. The fluorescence spectra of oxidized and reduced Zn-NIR are presented in Figure 1; the fluorescence, at all excitation wavelengths, is considerably greater in the reduced enzyme, and removal of oxygen is also associated with a small increase in the emission.

\section{Titration of apo-NIR with ANS}

It is well known that binding of ANS to proteins is associated with a large increase in the quantum yield of the dye and a blue shift in the emission maximum, e.g. upon binding to apo-

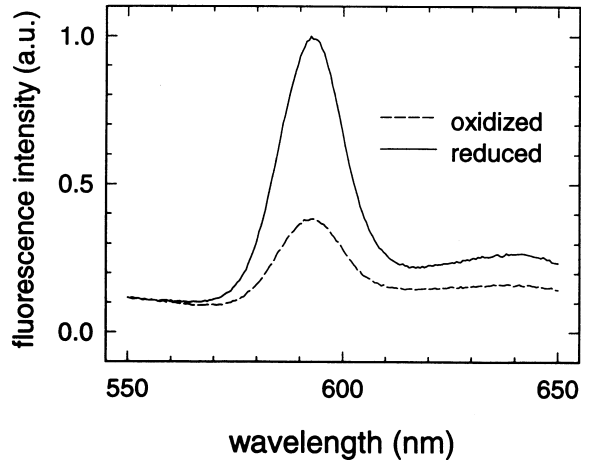

Figure 1 Fluorescence spectra of oxidized and reduced $\mathrm{Zn}-\mathrm{NIR}$

Conditions: $0.05 \mathrm{M}$ sodium phosphate buffer, $\mathrm{pH} 7.3$, temperature $25^{\circ} \mathrm{C}$, light path $1 \mathrm{~cm}$. Continuous line, reduced enzyme; broken line, oxidized enzyme. Zn-NIR concentration $0.63 \mu \mathrm{M}$; excitation wavelength $424 \mathrm{~nm}$.

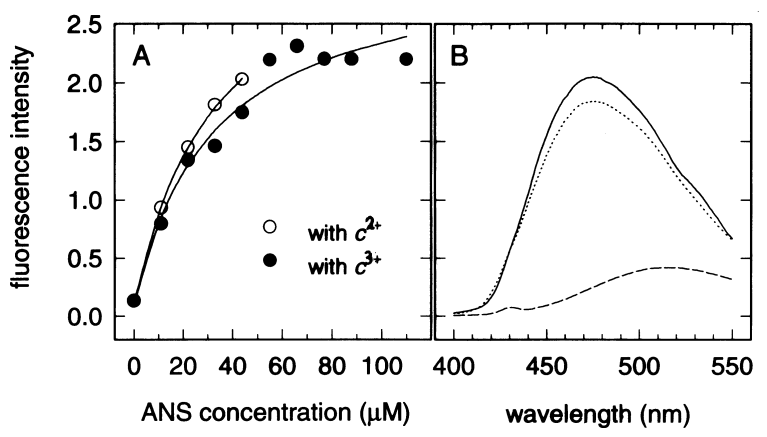

Figure 2 (A) Titration of apo-NIR (4.4 $\mu \mathrm{M} /$ monomer) with ANS, followed by fluorescence emission at $470 \mathrm{~nm}$ and (B) emission spectrum of (bound) ANS, excited at $375 \mathrm{~nm}$

(A) Open symbols: apo-NIR with ferrous c-haem; closed symbols: apo-NIR with ferric c-haem. (B) The 1-cm-pathlength cuvette contained $4.4 \mu \mathrm{M}$ apo-NIR and $45 \mu \mathrm{M}$ ANS dissolved in $0.05 \mathrm{M}$ sodium phosphate buffer, pH 7.4. Continuous line, NIR with ferrous c-haem; dotted line, NIR with ferric c-haem (obtained by addition of $20 \mu \mathrm{M}$ potassium ferricyanide); the broken line is the emission spectrum of $45 \mu \mathrm{M}$ free ANS in the same buffer.

myoglobin the quantum yield increases from 0.004 to 0.98 and the maximum emission shifts from 515 to $454 \mathrm{~nm}$ [16]. In NIR the blue shift of bound ANS is smaller and the fluorescence peaks at $470 \mathrm{~nm}$ (Figure 2B). Titration with ANS of reduced and oxidized apo-NIR (Figure 2A, open and closed symbols respectively) yields a dissociation constant $\left(K_{\mathrm{D}}=30 \mu \mathrm{M}\right)$ independent of the redox state of the c-haem. Moreover oxidation of the c-haem by ferricyanide causes only a marginal reduction of the emission spectrum of the adduct (Figure 2B). Bound ANS was (partially) displaced upon addition of Fe-protoporphyrin IX, which was suggested to bind at the $\mathrm{d}_{1}$-haem pocket [15].

\section{Dynamic absorption and fluorescence}

The time course of fluorescence decay of the Zn-NIR excited at $424 \mathrm{~nm}$ was measured with a Spex energy-modulated spectrofluorimeter, using fluorescein as a standard. This process is the fastest observed in this study, with a time constant of $2.4 \mathrm{~ns}$, and its rate constant agrees well with that measured for $\mathrm{Zn}$-cytochrome $c$ [9].

Flash-induced absorbance changes after excitation at $532 \mathrm{~nm}$ 


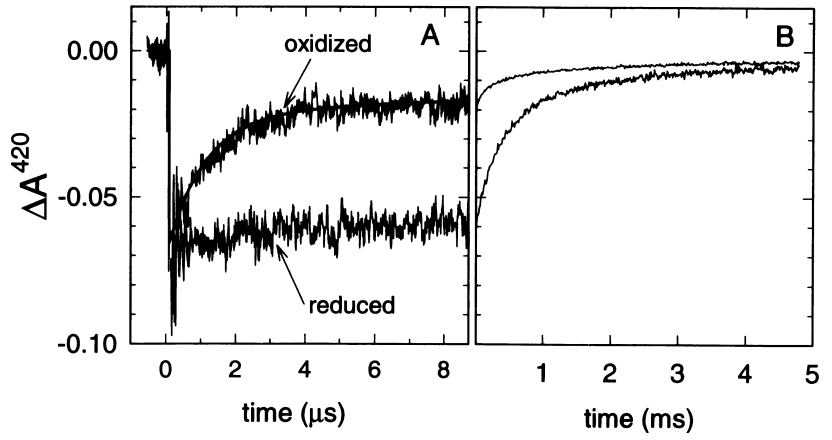

Figure 3 Time course of absorbance changes at $420 \mathrm{~nm}$ following photoexcitation of reduced and oxidized Zn-NIR (6.3 $\mu \mathrm{M})$ with a laser pulse

(A) and (B) refer to two different time scales.

are shown in Figure 3. The duration of the laser flash $(8 \mathrm{~ns})$ and the time constant of the detector $(50 \mathrm{~ns})$ preclude the observation of the first excited state, the fluorescent $\mathrm{Zn}$-porphyrin singlet. As demonstrated by Vanderkooi and co-workers [9] in Zn-substituted cytochrome $c$, the quantum yield of fluorescence is low $(<0.1)$ because of the rapid conversion of the singlet into a triplet state; this is the first intermediate which we observe (see also [12]).

It is difficult to estimate to what extent the excited state is populated, given the energy and duration of the pulse. Under our experimental conditions the maximal laser power was over 10fold higher than that required to yield the maximum absorbance change, and therefore, to avoid photochemical damage of the sample, the lowest energy level able to induce the full optical transition was used. Since fluorescence lifetime is shorter than the flash, the yield of the triplet state is increased because of reexcitation of the molecules already decayed to the ground state by fluorescence; moreover, according to Dixit et al. [9], in $\mathrm{Zn-}$ cytochrome $c$ the yield of triplet is 0.9 and exceeds that of fluorescence by over 10-fold. Thus, assuming that in Zn-NIR the rate of triplet formation from the singlet equals or exceeds that for fluorescence, we conclude that the yield of the triplet at the end of the $8 \mathrm{~ns}$ laser pulse is higher than 0.75 .

In the reduced $\mathrm{Zn}-\mathrm{NIR}$ (ferrous c-haem), the triplet state

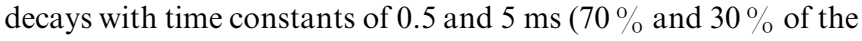
optical change observed at $420 \mathrm{~nm}$ respectively); these processes are observed as an increase in absorbance in Figure 3(B). These relatively slow reactions have been tentatively attributed to thermal as well as radiative (delayed fluorescence and phosphorescence) decay of the triplet excited state, following the interpretation of the data reported for $\mathrm{Zn}$-substituted cytochrome $c$ [9]. Note that these are apparent rate constants and correspond to the sum of the intrinsic rate constants describing each process which deactivates the triplet state.

On the other hand when the c-haem of Zn-NIR is oxidized (ferric c-haem) a rapid increase in absorbance with a relaxation time of about $1.4 \mu \mathrm{s}$ is observed (Figure 3A); this process is attributed to the rapid deactivation of the triplet excited state by eT from the $\mathrm{Zn}$ triplet to the ferric c-haem iron, producing an intermediate with $\mathrm{Fe}^{2+}$ c-haem and $\mathrm{Zn}$-porphyrin ${ }^{+}$. This intermediate is unstable, being almost undetectable in the kinetic difference spectrum (see below); thus we postulate that the electron is rapidly back transferred to the $\mathrm{Zn}$-porphyrin $^{+}$to restore the ground-state Zn-NIR. A contribution (25\%) to the recovery of the ground state is accounted for by slower processes
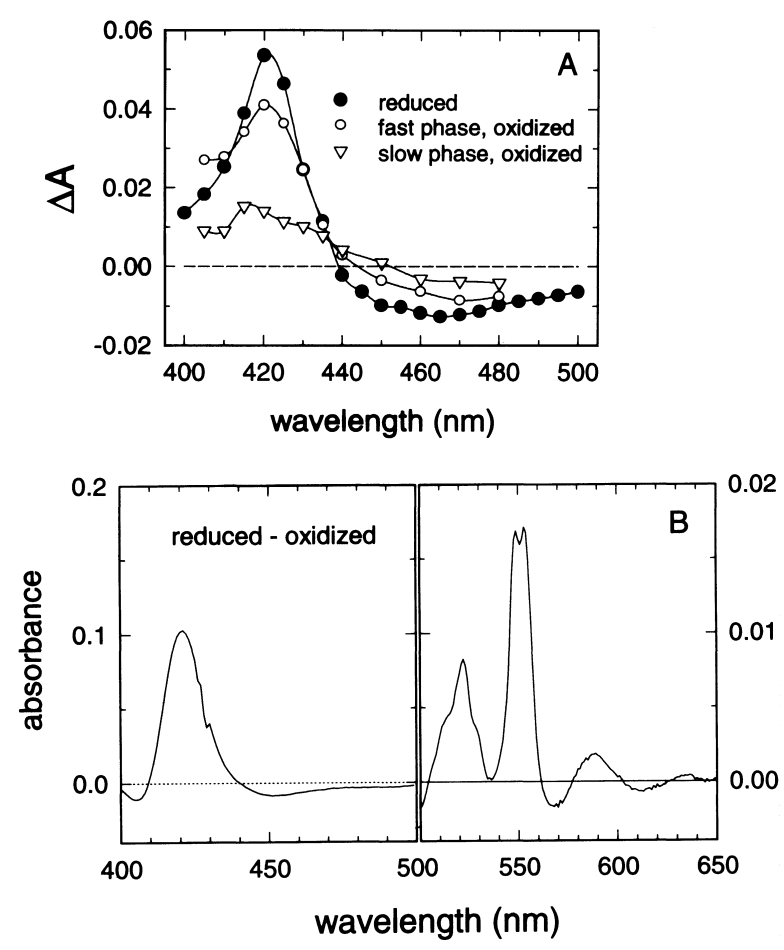

Figure 4 Kinetic and static difference spectra

(A) Kinetic difference spectra corresponding to the amplitude of the slow phase ( , reduced Zn-NIR; $\nabla$, oxidized Zn-NIR) and of the fast kinetic phase $(O$, oxidized Zn-NIR). As demonstrated in Figure 3, no fast kinetic phase is observed with reduced Zn-NIR. (B) Static difference spectrum of $6.3 \mu \mathrm{M}$ ferrous-ferric Zn-NIR

with rate constants similar to those observed in the reduced enzyme (Figure 3B); we attribute this observation to a fraction of the Zn-NIR which contains more than one Zn-porphyrin per monomer (see the Materials and methods section).

The kinetic difference spectra recorded for the oxidized and reduced derivatives of $\mathrm{Zn}-\mathrm{NIR}$, reported in Figure 4(A), are similar, but not identical; moreover they differ from the static oxidized-minus-reduced difference spectrum (reported in Figure 4B for comparison), a demonstration that throughout the time course the difference spectrum of the triplet state of the $\mathrm{Zn}$ porphyrin predominates; therefore starting with the oxidized enzyme the population of the $\mathrm{Fe}^{2+}-\mathrm{Zn}$-porphyrin ${ }^{+}$intermediate produced by eT contributes to a minor extent (as further discussed below).

The $1.4 \mu$ s process observed in oxidized $\mathrm{Zn}$-NIR was investigated at temperatures ranging from 1 to $35^{\circ} \mathrm{C}$, and was found to be temperature-independent in rate and amplitude; moreover the rate is the same at $\mathrm{pH}=7.3$ and $\mathrm{pH}=8.8$.

\section{DISCUSSION}

Based on the model proposed by Vanderkooi and co-workers [9], Figure 5 shows an energy-level diagram for the different states. Following the laser pulse, the $\mathrm{Zn}$-porphyrin is excited to a singlet state $2 \mathrm{eV}$ above the ground state. The reactions that depopulate the singlet state (with a measured lifetime of $2.4 \mathrm{~ns}$ ) are fluorescence, thermal conversion and population of the longlived triplet state. This in turn may decay through several paths: conversion of the triplet into the singlet state (which leads to the delayed fluorescence [9]), thermal dissipation, phosphorescence and, in the oxidized enzyme only, eT to the ferric iron of the c- 


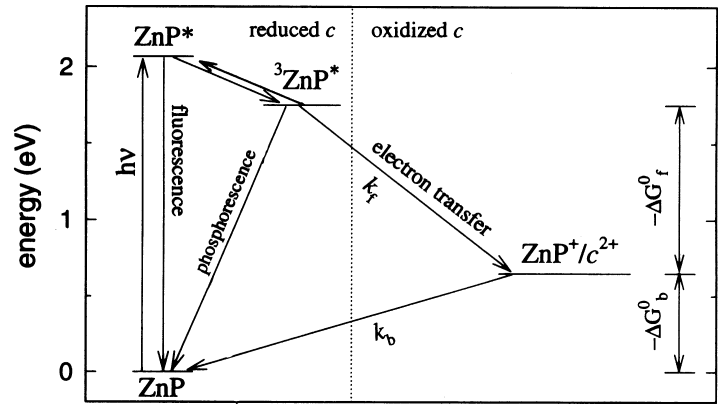

Figure 5 Energy levels of the Zn-porphyrin intermediates of the reaction pathway

Notice that the state $\mathrm{Zn}$-porphyrin ${ }^{+} / \mathrm{Fe}^{2+}$ is only accessible when the starting material is oxidized Zn-NIR.

haem, similarly to what is observed for the $1: 1$ complex of $\mathrm{Zn}$ cytochrome $c$-cytochrome $c$ oxidase [12].

The $1.4 \mu \mathrm{s}$ process (Figure 3) is attributed to eT rather than quenching through energy transfer for the following reasons: (i) it is not observed in the reduced enzyme; (ii) the overlap between the fluorescence spectrum and the absorption spectrum is small; in addition, the (integrated) absorption in the reduced state is slightly larger than in the oxidized state which would imply a larger degree of energy transfer in the former, contrary to what we observe; (iii) titration of the two redox states of apo-NIR with ANS demonstrates (Figure 2) that energy transfer between ANS bound to the $d_{1}$-haem pocket and the c-haem is only marginally influenced, if at all, by the redox state of the chaem. Thus, we conclude that the conformational change coupled to oxidation, seen by others [6,17], is unlikely to affect the extent of energy transfer from the Zn-porphyrin to the c-haem. Finally, the measured relaxation time of $1.4 \mu \mathrm{s}$ is consistent with comparable results on the Zn-cytochrome $c$-cytochrome $c$ oxidase complex [12].

The millisecond processes seen in reduced Zn-NIR (Figure 3) are implicitly assigned to thermal, delayed fluorescence and phosphorescence decay of the triplet state. A fraction ( $25 \%$ ) of these slower processes, seen also in the oxidized Zn-NIR, is attributed to a population of the enzyme containing an excess of bound $\mathrm{Zn}$-porphyrin, probably outside the $\mathrm{d}_{1}$-haem pocket.

Delayed fluorescence of the $\mathrm{Zn}$-porphyrin is due to conversion of the triplet into the singlet state [9]; thus steady-state fluorescence emission in the reduced Zn-NIR includes delayed as well as immediate fluorescence, while in the oxidized enzyme only immediate fluorescence makes a contribution, the delayed one being completely quenched by eT. From Figure 1 it is easy to estimate that in reduced Zn-NIR delayed fluorescence exceeds immediate fluorescence by approximately twofold, a result suggesting that the conversion of the triplet into the singlet is at least two times faster than the sum of phosphorescence and thermal decay. The pertinent rate constants are estimated at $4.2 \times 10^{8} \mathrm{~s}^{-1}$ for the sum of all processes leading to the decay of the singlet excited state and $2000 \mathrm{~s}^{-1}$ and $7 \times 10^{5} \mathrm{~s}^{-1}$ for those leading to the decay of the triplet state in the reduced and oxidized Zn-NIR respectively.

The very large difference in fluorescence yield between the oxidized and reduced states of Zn-NIR, which demonstrates a large contribution of delayed fluorescence, contrasts with the relatively low yield of delayed fluorescence in $\mathrm{Zn}$-cytochrome $c$ [9]; this is perhaps the most significant difference in the properties of reduced Zn-NIR compared with Zn-cytochrome $c$, and indicates a more efficient thermal conversion of the excited states of the Zn-porphyrin in NIR than in cytochrome $c$.

In the homologous NIR from Thiosphaera pantotropha, the c- $d_{1}$ haem-to-haem distance determined from the crystallographic co-ordinates of the oxidized enzyme is $11 \AA$ [2]. From the three-dimensional structure of Ps. aeruginosa NIR (C. Cambillau, M. Tegoni, M. C. Silvestrini and M. Brunori, unpublished work), the corresponding distance is $9 \AA$. The internal $\mathrm{eT}$ rate constant measured in this work with $\mathrm{Zn}$-NIR $\left(7.0 \times 10^{5}\right.$ $\mathrm{s}^{-1}$ ), even after correction for the large driving force, is still much higher (over 100-fold) than that determined by stopped flow $\left(k=0.3 \mathrm{~s}^{-1}\right)$ using reduced azurin as electron donor [7]. Such a slow rate is not justified by the haem-to-haem distance, as already pointed out by Fulop et al. [2].

The $\mathrm{d}_{1}$-haem iron is known to be hexaco-ordinated in the oxidized state [17]; in NIR from Thiosphaera pantotropha the sixth co-ordination ligand of $\mathrm{Fe}^{3+}$ is $\mathrm{Tyr}-25$ [2]. Since spectroscopy and ligand binding data indicate that the iron atom of the $\mathrm{d}_{1}$ haem is pentaco-ordinated in the reduced state, the substantial change in co-ordination of the $\mathrm{d}_{1}$-haem upon reduction may possibly account for the very slow rate of internal eT seen by stopped flow, given that the reorganization energy term of Marcus and Sutin [18] would contribute substantially to slow down the eT rate; no similar rearrangement is expected to occur in the experiments described in this work.

We are indebted to Dr. Sergio Costantini (Istituto Superiore di Sanità, Roma, Italy) for the atomic absorption spectroscopy measurements, and to Robert Sjoback and Nicke Svanvik (Goteborg University, Goteborg, Sweden) for fluorescence relaxation measurements. This work was partially supported by the Progetto Strategico 'Molecular Manufactoring', C.N.R., Italy, by the M.U.R.S.T. of Italy $(40 \%$, 'Liveprotein') and by grants from the Swedish Natural Science Research Council, the Knut and Alice Wallenberg Foundation, the Magn. Bergvalls Foundation and the Helge Ax son Johnsons Foundation.

\section{REFERENCES}

1 Yamanaka, T., Ota, A. and Okunuki, K. (1961) Biochim. Biophys. Acta 53, 294-308

2 Fulop, V., Moir, J. W. B., Ferguson, J. S. and Hajdu, J. (1995) Cell 81, 369-377

3 Chang, C. K., Timkovich, R. and Wu, W. (1986) Biochemistry 25, 8447-8453

4 Silvestrini, M. C., Falcinelli, S., Ciabatti, I., Cutruzzolà, F. and Brunori, M. (1994) Biochimie 76, 641-654

5 Johnson, M. K., Thomson, A. J., Walsh, T. A., Barber, D. and Greenwood, C. (1980) Biochem. J. 189, 285-294

6 Sutherland, J., Greenwood, C., Peterson, J. and Thomson, A. J. (1986) Biochem. J. 233, 893-898

7 Parr, S. R., Barber, D., Greenwood, C. and Brunori, M. (1977) Biochem. J. 167, 447-455

8 Greenwood, C., Barber, D., Parr, S. R., Antonini, E., Brunori, M. and Colosimo, A. (1978) Biochem. J. 173, 11-17

9 Dixit, S. N., Moy, V. T. and Vanderkooi, J. M. (1984) Biochemistry 23, 2103-2107

10 McGourty, J. L., Blough, N. V. and Hoffman, B. M. (1983) J. Am. Chem. Soc. 105 4470-4472

11 Zhou, J. S. and Kostic, N. M. (1993) Biochemistry 32, 4539-4546

12 Brzezinski, P., Sundhal, M., Adelroth, P., Wilson, M. T., El-Agez, B., Wittung, P. and Malmstrom, B. G. (1995) Biophys. Chem. 54, 191-197

13 Silvestrini, M. C., Cutruzzolà, F., D'Alessandro, R., Brunori, M., Fochesato, N. and Zennaro, E. (1992) Biochem. J. 285, 661-666

14 Hallen, S. and Brzezinski, P. (1994) Biochim. Biophys. Acta 1184, 204-218

15 Hill, K. E. and Wharton, D. C. (1978) J. Biol. Chem. 253, 489-495

16 Stryer, L. (1965) J. Mol. Biol. 13, 482-495

17 Walsh, T. A., Johnson, M. K., Greenwood, C., Barber, D., Springall, J. P. and Thomson, A. J. (1979) Biochem. J. 177, 29-39

18 Marcus, R. A. and Sutin, N. (1985) Biochim. Biophys. Acta 811, 265-322 